\title{
Percutaneous Spinal Cord Stimulation for Failed Back Surgery Syndrome: A Retrospective Study
}

\author{
Ferhat HARMAN ${ }^{1,2}$, Seckin AYDIN³, Savas SENCAN4, Esra AKDENIZ ${ }^{5}$, Yahya GUVENC ${ }^{1,2}$, Ayten SARACOGLU ${ }^{6}$, \\ Can EYIGOR ${ }^{7}$, Meltem UYAR ${ }^{7}$, Osman Hakan GUNDUZ ${ }^{4}$ \\ ${ }^{1}$ Marmara University, School of Medicine, Department of Neurosurgery, Istanbul, Turkey \\ ${ }^{2}$ Marmara University Institute of Neurological Sciences, Department of Neurosurgery, Istanbul, Turkey \\ ${ }^{3}$ University of Health Sciences, Okmeydani Training and Research Hospital, Department of Neurosurgery, Turkey \\ ${ }^{4}$ Marmara University, School of Medicine, Department of Physical Medicine and Rehabilitation, Division of Pain Medicine, Istanbul, Turkey \\ ${ }^{5}$ Marmara University, School of Medicine, Department of Medical Education, Istanbul, Turkey \\ ${ }^{6}$ Marmara University, School of Medicine, Department of Anesthesiology and Reanimation, Istanbul, Turkey \\ ${ }^{7}$ Ege University, School of Medicine, Department of Anesthesiology and Reanimation, Division of Pain Medicine, Izmir, Turkey
}

Corresponding author: Ferhat HARMAN ferhatharman@hotmail.com

\section{ABSTRACT}

AIM: To evaluate the outcomes of percutaneous spinal cord stimulation (PSCS) in patients with failed back surgery syndrome (FBSS) in an academic tertiary care center.

MATERIAL and METHODS: The hospital records of patients with FBSS who had undergone PSCS were retrospectively reviewed. A total of 19 patients with FBSS matched the search criteria, and among them, 16 were included in the study, in whom permanent implantable pulse generators (IPGs) were implanted. Demographic, clinical and surgical outcomes were evaluated.

RESULTS: Twelve (75\%) women and 4 (25\%) men with a median age of 50 years (range, 35-80 years) were analysed. The average number of surgeries before PSCS was $1.6 \pm 1.2$ (range, 1-4). Pain was localised in the back and leg in $81.25 \%$ of the patients. The mean duration of symptoms was $6.3 \pm 3.1$ years (range, $2-10$ years). The mean length of trial period was $16.3 \pm 6.8$ days (range, $7-29$ days). In this study, the permanent implantation rate was $84.2 \%(16 / 19)$. The mean follow-up time was $18.3 \pm 3.9$ months (range, 14-26 months). Postoperative back/leg numerical pain rating scale (NPRS) score was significantly lower than preoperative back/ leg NPRS score $(p<0.001)$. The postoperative Oswestry Disability Index (ODI) score was significantly lower than the preoperative ODI score $(p<0.001)$.

CONCLUSION: PSCS is a safe and effective treatment method for patients with FBSS. In this study, the high rate of improvement in the outcome scores may be attributed to the small sample size and early PSCS implantation.

KEYWORDS: Failed back surgery syndrome, Chronic pain, Spinal cord stimulation, Minimally invasive surgery

ABBREVIATIONS: SCS: Spinal cord stimulation, FBSS: Failed back surgery syndrome, PSCS: Percutaneous spinal cord stimulation, EPG: External pulse generator, IPG: Implantable pulse generator, ODI: Oswestry Disability Index, NPRS: Numerical pain rating scale, IQR: Interquartile range, PP: Percentile-percentile, QQ: Quantile-quantile, MRI: Magnetic resonance imaging

Ferhat HARMAN (1) : 0000-0003-4685-2201

Seckin AYDIN (1): 0000-0003-1195-9084

Savas SENCAN (1) : 0000-0001-8150-0581
Esra AKDENIZ (1) : 0000-0002-3549-5416

Yahya GUVENC : : 0000-0002-4813-0854

Ayten SARACOGLU (1): 0000-0002-1186-0933
Can EYIGOR

Meltem UYAR

(1) : 0000-0002-7991-8564

Osman Hakan GUNDUZ (10) : 0000-0002-3214-803X 


\section{- INTRODUCTION}

S pinal cord stimulation (SCS) has been an effective modality for the management of chronic pain with several aetiologies for a long time $(7,26)$. Likewise, it is also a preferable treatment option for failed back surgery syndrome (FBSS), which is a challenging pathology in neurosurgical practice $(28,30)$. The main goal of treatment is to decrease chronic back and/or leg pain, to mobilise and to increase the quality of life.

Recently, minimally invasive approaches have begun to be implemented more frequently, and percutaneous spinal cord stimulation (PSCS) is one of these methods. Although the efficiency of this modality has been proven so far, the area of use is still limited considering that it is an invasive intervention $(8,12,27)$. High success and low complication rates are obtained depending on technological advances and surgeons' surgical experience (24). This study reviews the characteristics and outcomes of PSCS in patients with chronic pain secondary to a neurosurgical procedure such as FBSS.

\section{MATERIAL and METHODS}

\section{Patients}

Patients undergoing PSCS implantation between January 2016 and December 2018 were reviewed retrospectively. This study was conducted according to the ethical standards of the Declaration of Helsinki of the Word Medical Association. All patients were informed about the risks of the treatment and the probability of treatment success. Informed consent forms were obtained.

Patients with FBSS who had a history of at least one spinal operation with postoperative chronic back and/or leg pain and who had unsuccessful conservative (medical and physiotherapy) treatments were included in this study. All patients underwent consultations for algology, physical therapy and rehabilitation. Neuropsychiatric evaluations were performed for all patients. To evaluate epidural fibrosis, preoperative contrast-enhanced thoracolumbar magnetic resonance imaging (MRI) was performed for all patients. Patients who have not had epidural fibrosis below the L3 level on MRI were chosen to undergo PSCS. Those requiring any surgical treatment were excluded from the study. Demographic characteristics, medical history and localisation and duration of pain were recorded and analysed.

\section{Technique}

Preoperative contrast-enhanced thoracolumbar MRI was performed for all patients. The patients were placed in the prone position on the radiolucent operation table. Intravenous (IV) cefazolin $30 \mathrm{mg} / \mathrm{kg}$ was administered before the surgery for prophylaxis. Under mild sedation, local anaesthesia was applied to the small skin incision site for the percutaneous electrodes. The percutaneous electrodes were inserted into the epidural space through the needle guide with fluoroscopy via a thoracolumbar paramedian approach in a sterile fashion. To apply the technique safely by needle tip and without injuring the spinal cord, the authors chose the lowest entry points as possible. If the electrode placement is aimed at the T8-T10 level, the L1-L2 level was the entry point, and if electrode placement is aimed at the T9-T11 level, the entry point was the L2-L3 level. According to the area and distribution of the patients' pain, the levels covered by the electrodes were planned and the number of electrodes was thus determined. Consequently, single or dual linear percutaneous electrodes were placed. The same procedure was repeated on the contralateral side for patients who received dual linear percutaneous electrodes. Paraesthesia coverage was determined by stimulation. Leads were then linked to an external pulse generator (EPG) by an extension cable. This extension cable was externalised approximately $15 \mathrm{~cm}$ away from the spinous process, which was on the opposite side of the patients' dominant hand.

The patients were then observed after discharge or stayed in the hospital throughout the trial period. At the end of the trial period, pain was evaluated, and the patients who benefited from the procedure were prepared for permanent implantable pulse generator (IPG) placement. Under local anaesthesia, the patients were placed in the prone position in a sterile fashion. After IV antibiotic prophylaxis, a subdermal pouch was placed on the opposite side of the EPG on the superior gluteal region for the permanent placement of the IPG. The extension cable of the electrodes was connected to the IPG subcutaneously.

\section{Outcome Assessment}

The patients were evaluated using the Oswestry Disability Index (ODI) and numerical pain rating scale (NPRS) (back and/or leg) preoperatively and 12 months after the operation. NPRS scores ranged from 0 (no pain at all) to 10 (highest level of pain), and ODI scores ranged from $0 \%$ to $100 \%$ (scores are stratified based on the severity of disability).

\section{Decision of Permanent IPG Placement}

An NPRS (back and/or leg) was used to decide whether to perform permanent IPG placement or not. At the end of the trial period, the patients were asked if the severity of pain decreased. If a patient described at least $50 \%$ of reduction in pain, the trial was considered to be successful, so permanent IPG placement is then performed. The patients whose pain did not improve at the end of the trial period were excluded from the study. The follow-up was performed annually through outpatient visits.

\section{Statistical Analysis}

Power calculations were based on an estimated effect size of 0.8 from (15) and on the proposed biological plausibility that ODI scores were assumed to be lower in the postoperative period than in the preoperative period. Thus, for a one-sided test, with a significance level of 0.05 (confidence limit 95\%) and power of 0.8 , approximately 13 participants were required to assume a non-normal parent distribution (5).

The normality of the numeric variables (age, number of operations, length of trial, duration of follow-up, and differences between the preoperative and postoperative back/leg NPRS and ODI scores) was assessed using percentile-percentile $(\mathrm{PP})$ and quantile-quantile (QQ) plots, skewness and kurtosis 
values and the Shapiro-Wilk test. The numeric variables were presented in means and standard deviations if the normality assumption was met and in median and interquartile range (IQR) if the minimum-maximum values were provided in both cases. The exact Wilcoxon-Pratt signed-rank test was used because of its robust power to compare dependent variables between two groups independently of their distributions.

For the analyses of paired groups, Gardner-Altman estimation plots visualising the effect sizes were given (10). The correlations between preoperative NPRS and ODI scores, as well as the comparison between postoperative NPRS and ODI scores, were assessed using Spearman's rank correlation coefficient. This was done by measuring the consistency of the pain scales with each other. The Wilcox sign test function in the R package coin was used for the exact calculation of the $p$ values of the Wilcoxon-Pratt signed-rank test. Statistical analyses were performed using the $\mathrm{R}$ statistical software, version 3.6.2 (The R Foundation, Vienna, Austria). The power calculations were conducted using $\mathrm{G}^{*}$ Power 8 (HeinrichHeine-Universität Düsseldorf, Düsseldorf, Germany). All $p$ values were one-sided, and a $p$ value of $<0.05$ was considered statistically significant.

\section{RESULTS}

Permanent IPGs were placed into 16 of 19 patients with FBSS. Three patients were excluded from the study because the pain reduction following PSCS was unsatisfactory. Their ages ranged from 35 to 80 years with a median of 50 years. There were $12(75 \%)$ women and $4(25 \%)$ men. While all patients underwent at least one lumbar spinal surgery, the average number of operations before PSCS was $1.6 \pm 1.2$ (range, 1-4). Pain was localised in the back and leg in 13 patients (81.25\%). Pain was bilateral in 11 patients $(68.7 \%)$ and unilateral in five patients (31.3\%). Lead levels were at T8-T10 in 10 patients (62.5\%) and at T9-T11 in 6 patients (37.5\%) (Table I). The mean duration of symptoms was $6.3 \pm 3.1$ years (range, $2-10$ years). The mean duration of the trial was $16.3 \pm 6.8$ days (range, 7-29 days). The mean follow-up duration was $18.3 \pm$ 3.9 months (range, 14-26 months).

Preoperative and postoperative ODI and NPRS scores were compared, and the Wilcoxon-Pratt signed-rank test results are shown in Table II. According to these statistical results, the mean postoperative back NPRS score was significantly lower than the mean preoperative back NPRS score (2 (range, $1-6)$ vs. 8 (range, $6-10$ ); $p<0.001$ ). The mean postoperative leg NPRS score was significantly lower than the mean preoperative leg NPRS score (2 (range, 1-7) vs. 8 (range, 6-10); $p<0.001$ ) (Figure 1A, B). The mean postoperative ODI score was significantly lower than the mean preoperative ODI score (16 (range, 0-52) vs. 85 (range, 70-100); $p<0.001$ ) (Figure 2). More than $50 \%$ reduction in the back/leg NPRS scores was observed in all patients who received permanent SCS.

Two serious complications occurred in one patient: CSF leakage and complex regional pain syndrome, which were due to the injury of the dorsal cord. This patient did not undergo permanent IPG placement.

\section{DISCUSSION}

In 1965, Melzack and Wall first introduced the gate control theory, which was related to the physiopathology of pain (19). Based on this theory, pain suppression was achieved by performing dorsal column stimulation (25). This treatment method, called the spinal cord stimulator, has been used for a long time to reduce chronic, intractable pain associated with various aetiologies (11).

FBSS, which is characterised by back and leg pain that does not improve after spinal surgery, limits patients' physical

Table I: Demographics, Clinical and Spinal Cord Stimulation (SCS) Characteristics of 16 Patients with Failed Back Surgery Syndrome (FBSS) Patients

FBSS patients $(n=16)$

\section{Demographic features}

Gender, n(\%)

\begin{tabular}{cc}
\hline \multicolumn{1}{c}{ Male } & $4(25.0)$ \\
\hline \multicolumn{1}{c}{ Female } & $12(75.0)$ \\
\hline Age $^{*}$ (years), mm-max & $50(35-80)$ \\
\hline
\end{tabular}

History, $\mathrm{n}(\%)$

\begin{tabular}{|c|c|}
\hline Laminectomy & $10(62.5)$ \\
\hline Laminectomy + instrumentation & $6(37.5)$ \\
\hline Number of previous lumbar operations $*$ & $1.6 \pm 1.2(1-4)$ \\
\hline \multicolumn{2}{|l|}{ Clinical features } \\
\hline Duration of symptoms ${ }^{*}$ (years) & $6.3 \pm 3.1(2-10)$ \\
\hline \multicolumn{2}{|l|}{ Localisation of pain } \\
\hline Only back & $2(12.5 \%)$ \\
\hline Back and leg & $13(81.25 \%)$ \\
\hline Only leg & $1(6.3 \%)$ \\
\hline
\end{tabular}

Side, $\mathrm{n}(\%)$

\begin{tabular}{lr}
\hline Unilateral & $5(31.3)$ \\
\hline Bilateral & $11(68.7)$ \\
\hline
\end{tabular}

Lead Levels, $n(\%)$

T8-10 $10(62.5)$

T9-11 $6(37.5)$

Trial period ${ }^{*}$ (days) $16.3 \pm 6.8(7-29)$

Permanent SCS rate $84.2 \%(16 / 19)$

Follow-up ${ }^{*}$ (months) $18.3 \pm 3.9(14-26)$

*: median, $¥:$ mean \pm standard deviation. 
Table II: Comparison of Pre and Postoperative Numerical Pain Rating Scale (NPRS) and Oswestry Disability Index (ODI) Scores

\begin{tabular}{|c|c|c|c|c|}
\hline & Preoperative & Postoperative & z (test-stat) & $\mathbf{p}$ \\
\hline $\begin{array}{l}\text { Leg NPRS, median (IQR) } \\
\text { range } \\
(n=14)\end{array}$ & $\begin{array}{c}8(7-10) \\
6-10\end{array}$ & $\begin{array}{c}2(2-4) \\
1-7\end{array}$ & 3.392 & $<0.001$ \\
\hline $\begin{array}{l}\text { ODI score, median (IQR) } \\
\text { range } \\
(n=16)\end{array}$ & $\begin{array}{c}85(80-92) \\
70-100\end{array}$ & $\begin{array}{c}16(13-35) \\
0-52\end{array}$ & 3.517 & $<0.001$ \\
\hline
\end{tabular}

IQR: Interquartile range; $p$ : Exact Wilcoxon-Pratt Signed-Rank Test one-sided p-value.

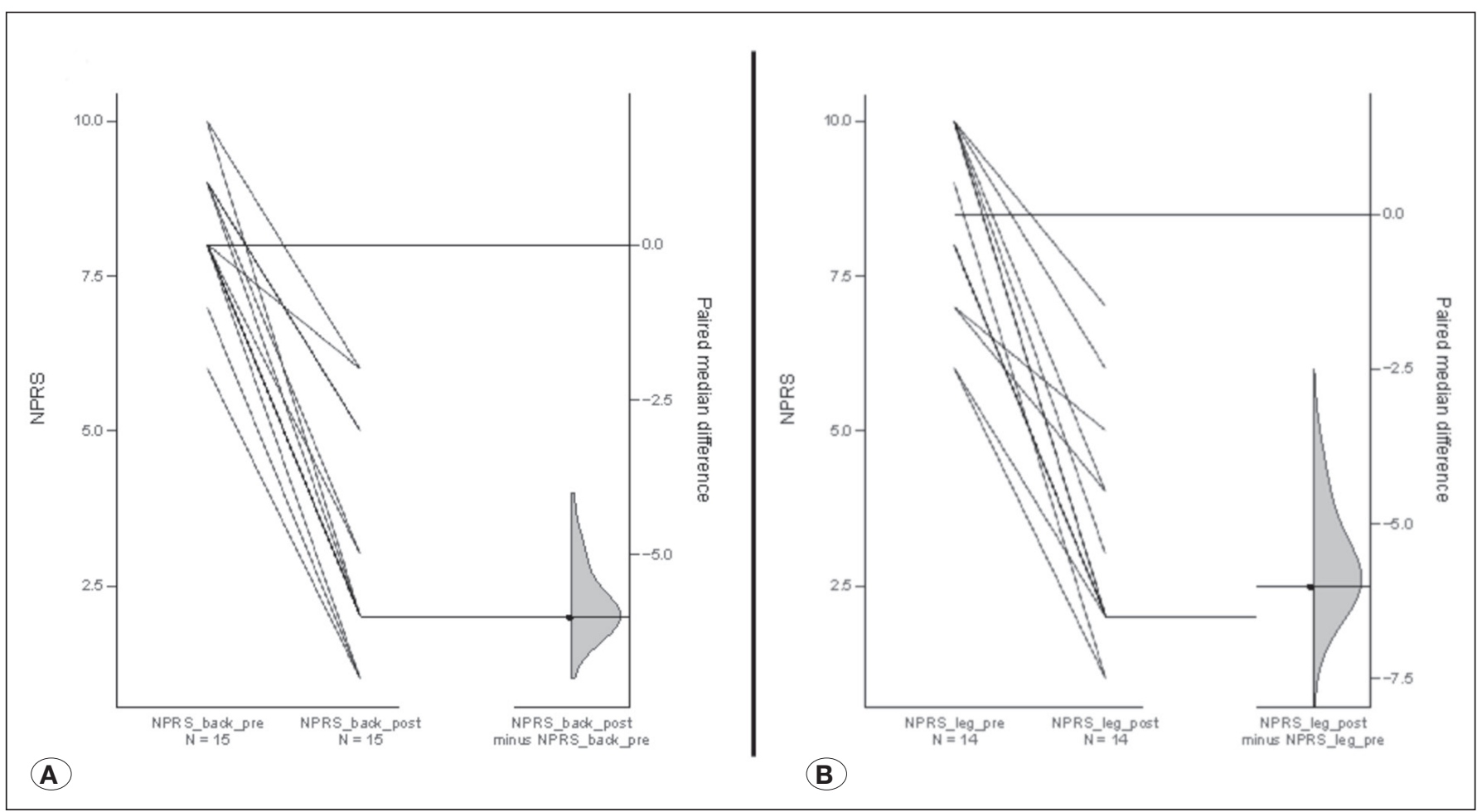

Figure 1: A) The left part of the estimation plots shows each patient's preoperative and postoperative back NPRS scores, where the dark line shows the median score of all patients. The right part of the plot shows the bootstrap distribution of the median differences with bias-corrected and accelerated 95\% confidence interval. B) The left part of the estimation plots shows each patient's preoperative and postoperative leg NPRS scores, where the dark line shows the median score of all patients. The right part of the plot shows the bootstrap distribution of the median differences with bias-corrected and accelerated $95 \%$ confidence interval.

activities and impairs sleep patterns. In other words, FBSS reduces patients' quality of life. The results obtained through medical treatments and new surgical interventions are not always satisfactory. SCS is a suitable, safe, and cost-effective treatment option for patients with FBSS (4).

SCS may be performed using percutaneous or conventional (e.g., open surgery and laminectomy/laminotomy) techniques. While the percutaneous technique is performed under local anaesthesia with the guidance of fluoroscopy, conventional techniques require general anaesthesia. Therefore, percutaneous placement of electrodes is less invasive, and the duration of operation is shorter (13). In addition, the area covered by paraesthesia can be accurately determined through communication with the awakened patient. In the percutaneous technique, although the postoperative pain is less, the risk of lead malposition is reported to be higher $(2 \%-22 \%)(1,18)$. In conventional techniques, lead positioning is more accurate because the surgeon directly exposes the lead placement site, and postoperative complication rates are significantly 


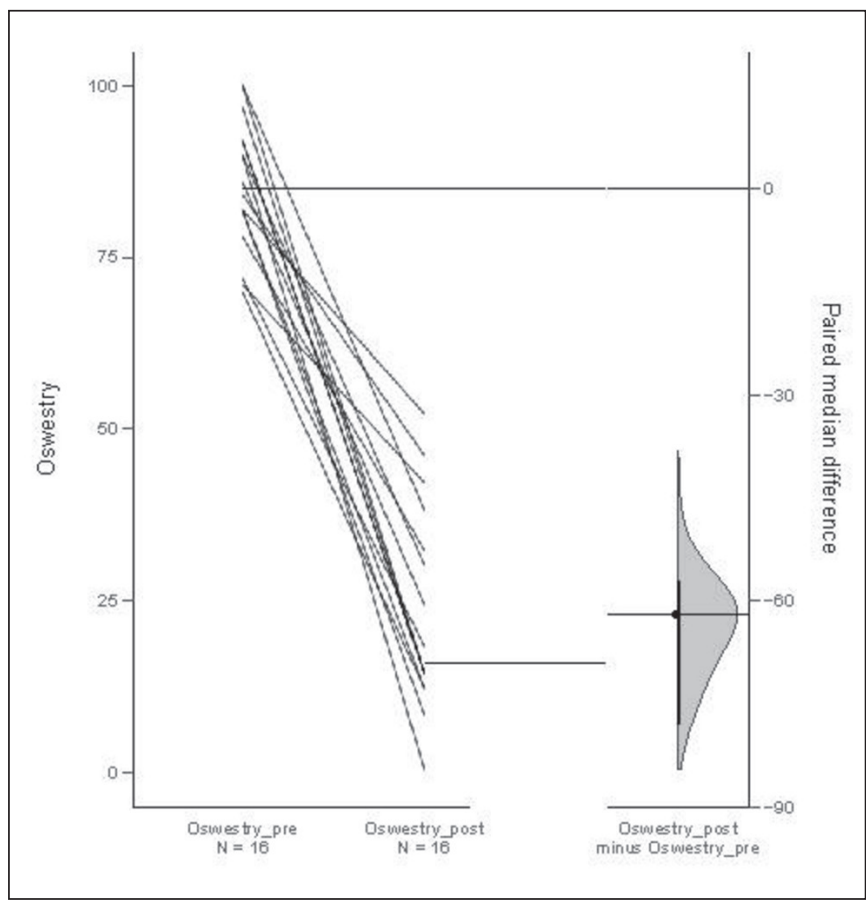

Figure 2: The left part of the estimation plot shows each patient's preoperative and postoperative ODI scores, where the dark line shows the median score of all patients. The right part of the plot shows the bootstrap distribution of the median differences with bias-corrected and accelerated 95\% confidence interval.

higher, while reoperation rates are lower $(1,13)$. Considering the literature and the results of this study, the percutaneous technique is a plausible treatment option for FBSS.

The patients underwent a trial with temporary leads placed to determine whether they can benefit or not from permanent SCS treatment. Although this trial process is widely applied, the difference between temporary and permanent implant applications remains uncertain. Kumar et al. reported that $80 \%$ of patients underwent permanent SCS placement after the trial (16). Alternatively, Turner et al. reported that permanent SCS placement was performed in $53 \%$ of patients in their case series (29). In another study, permanent SCS placement was performed in all patients after the trial (31). In the current study, the authors implanted permanent SCS to $84.2 \%$ of the patients after the trial. In this study, the average number of operations before SCS implantation was $1.6 \pm 1.2$ (range, $1-4)$, which is comparatively lower than that in the relevant literature (28). Lower fibrosis rates due to few operations may be a factor for explaining the high success rate of the trial in this study.

There is no definite information or advice about the duration of the trial in the literature. The optimum duration should be determined based on the patients' tolerance to temporary SCS, ensuring the response to treatment and avoiding infection risks. SCS studies with trials various durations, such as $3-30$ days, have been previously reported $(6,13,20)$. Although the trial duration in this study was indefinite, the average duration of the trial was 16 days.
In the literature review, SCS treatment provided significant pain relief in patients with FBSS. North et al. reported a randomised controlled series of patients with FBSS, and at least $50 \%$ pain reduction was detected in $47 \%$ of patients who received percutaneous SCS (20). Consequently, Kumar et al. reported over $50 \%$ pain reduction in $48 \%$ of 50 patients who had received SCS for FBSS treatment (16). Dario et al. reported favourable results; 21 of 23 patients had undergone permanent SCS placement (6). However, Ohnmeiss et al. reported that 40 patients who underwent SCS placement had $80 \%$ pain reduction in the short term and $70 \%$ in the long term (23). Cameron et al. stated that the overall success rate of eight prospective studies was $65 \%$ in the literature review regarding the effectiveness of SCS in treating chronic pain (3). In the current study, all patients had more than $50 \%$ reduction in their pain assessment scores at the last follow-up, and these results are distinctly higher compared with those in the literature.

In a case series of 40 patients, $85 \%$ of whom were diagnosed with FBSS, a statistically significant decrease was reported in the mean ODI score after SCS (2). Moreover, Kumar et al. reported similar results in their large case series (16). In the current study, postoperative back/leg NPRS and ODI scores were significantly lower than preoperative scores. In the authors' retrospective study on the outcomes of PSCS treatment, the authors assumed that the high success rate of pain reduction might be due to the limited number of patients.

Although SCS is mostly utilised to treat radicular or radiating pain, axial (low back) pain is more difficult to treat. This information is available as a 'level B' recommendation in the guidelines $(22,27)$. Kumar et al. examined the visual analogue scale scores in patients with FBSS treated with single-lead systems for low-back and leg pain. They reported that these patients had $15 \%$ and $64 \%$ reduction in axial pain and radicular pain, respectively (14). Similar results have been reported in other series $(17,21)$. In the current study, besides having a significant decrease in radicular pain, a significant reduction in axial pain was also achieved. Using dual leads and progress in programming technology may be factors for the satisfying results of axial pain reduction.

PSCS complications could be device-related or of biological origin. Device-related complications are more common, and more than half of the complications are observed within 6 months after the device has been placed (9). In the current case series, with an average follow-up period of 16 months, no device-related complications such as lead migration or device battery depletion were recorded. No patients had SCS-related infection. Only one patient developed complex regional pain syndrome and CSF leakage during the trial period.

One of the limitations of this study is its small sample size. While only the PSCS results in this study were examined, no comparative randomised study was conducted. The postoperative ODI and NPRS scores were measured only annually. These were not measured at regular intervals. The effect of SCS should have been measured and compared at the early and late postoperative periods to assess the decrease in efficiency. 


\section{- CONCLUSION}

Percutaneous SCS is a safe and effective treatment method for FBSS that has been used for a long time. Treatment effectiveness increased with the development of implanted devices with advancing technology and increasing surgical experience of surgeons. In this study, retrospective analyses were performed for patients who underwent PSCS in the authors' clinic, and successful results were obtained in accordance with the literature. The high rate of improvement in the outcome scores may be attributed to the small sample size and early PSCS implantation.

\section{ACKNOWLEDGMENT}

Preparation for publication of this article is partly supported by Turkish Neurosurgical Society.

\section{- REFERENCES}

1. Babu R, Hazzard MA, Huang KT, Ugiliweneza B, Patil CG, Boakye M, Lad SP: Outcomes of percutaneous and paddle lead implantation for spinal cord stimulation: A comparative analysis of complications, reoperation rates, and health-care costs. Neuromodulation 16:418-426, 2013

2. Burchiel KJ, Anderson VC, Wilson BJ, Denison DB, Olson KA, Shatin D: Prognostic factors of spinal cord stimulation for chronic back and leg pain. Neurosurgery 36:1118-1134, 1995

3. Cameron T: Safety and efficacy of spinal cord stimulation for the treatment of chronic pain: A 20-year literature review. J Neurosurg 100:254-267, 2004

4. Cho JH, Lee JH, Song KS, Hong JY: Neuropathic pain after spinal surgery. Asian Spine J 11:642-652, 2017

5. Cumming G: Understanding the New Statistics: Effect Sizes, Confidence Intervals, and Meta-Analysis. New York, NY: Routledge, 2012

6. Dario A, Fortini G, Bertollo D, Bacuzzi A, Grizzetti C, Cuffari $\mathrm{S}$ : Treatment of failed back surgery syndrome. Neuromodulation 4:105-110, 2001

7. Devulder J, De Colvenaer L, Rolly G, Caemaert J, Calliauw L, Martens F: Spinal cord stimulation in chronic pain therapy. Clin J Pain 6:51-56, 1990

8. Frey ME, Manchikanti L, Benyamin RM, Schultz DM, Smith HS, Cohen SP: Spinal cord stimulation for patients with failed back surgery syndrome: A systematic review. Pain Phys 12:379-397, 2009

9. Hayek SM, Veizi E, Hanes M: Treatment-limiting complications of percutaneous spinal cord stimulator implants: A review of eight years of experience from an academic center database. Neuromodulation 187:603-609, 2015

10. Ho J, Tumkaya T, Aryal S, Choi H, Claridge-Chang A: Moving beyond $P$ values: Data analysis with estimation graphics. Nat Methods 16:565-566, 2019

11. Kapural L: Spinal cord stimulation for intractable chronic pain. Curr Pain Headache Rep 18:406, 2014

12. Kelly GA, Blake C, Power CK, O'Keeffe D, Fullen BM: The impact of spinal cord stimulation on physical function and sleep quality in individuals with failed back surgery syndrome: A systematic review. Eur J Pain 16:793-802, 2012
13. Kinfe TM, Schu S, Quack FJ, Wille C, Vesper J: Percutaneous implanted paddle lead for spinal cord stimulation: Technical considerations and long-term follow-up. Neuromodulation 15:402-407, 2012

14. Kumar K, Hunter G, Demeria D: Spinal cord stimulation in treatment of chronic benign pain: Challenges in treatment planning and present status, a 22-year experience. Neurosurgery 58:481-496, 2006

15. Kumar K, Rizvi S, Bnurs SB: Spinal cord stimulation is effective in management of complex regional pain syndrome I: Fact or fiction. Neurosurgery 69:566-580, 2011

16. Kumar K, Taylor RS, Jacques L, Eldabe S, Meglio M, Molet J, Thomson S, O'Callagan J, Eisenberg E, Milbouw G, Buchser E, Fortini G, Richardson J, North RB: Spinal cord stimulation versus conventional medical management for neuropathic pain: a multicentre randomized controlled trial in patients with failed back surgery syndrome. Pain 132:179-188, 2007

17. Lang P: The treatment of chronic pain by epidural spinal cord stimulation - a 15 year follow up; present status. Axon 18:7173, 1997

18. Mekhail NA, Mathews M, Nageeb F, Guirguis M, Mekhail MN, Cheng J: Retrospective review of 707 cases of spinal cord stimulation: Indications and complications. Pain Pract 11:148153, 2011

19. Melzack R, Wall PD: Pain mechanisms: A new theory. Science 150: 971-979, 1965

20. North RB, Kidd DH, Farrokhi F, Piantadosi SA: Spinal cord stimulation versus repeated lumbosacral spine surgery for chronic pain: A randomized, controlled trial. Neurosurgery 56:98-106, 2005

21. North RB, Kidd DH, Zahurak M, James CS, Long DM: Spinal cord stimulation for chronic, intractable pain experience over two decades. Neurosurgery 32:384-395, 1993

22. North R, Shipley J: Practice parameters for the use of spinal cord stimulation in the treatment of chronic neuropathic pain. Pain Med 8:200-275, 2007

23. Ohnmeiss DD, Rashbaum RF, Bogdanffy GM: Prospective outcome evaluation of spinal cord stimulation in patients with intractable leg pain. Spine 21:1344-1350, 1996

24. Shamji MF, De Vos C, Sharan A: The advancing role of neuromodulation for the management of chronic treatmentrefractory pain. Neurosurgery 80:108-113, 2017

25. Shealy CN, Mortimer JT, Reswick JB: Electrical inhibition of pain by stimulation of the dorsal columns: Preliminary clinical report. Anesth Analg 46:489-491, 1967

26. Sitzman BT, Provenzano DA: Best practices in spinal cord stimulation. Spine (Phila Pa 1976) 42:67-71, 2017

27. Taylor RS, Desai MJ, Rigoard P, Taylor RJ: Predictors of pain relief following spinal cord stimulation in chronic back and leg pain and failed back surgery syndrome: A systematic review and meta-regression analysis. Pain Practice 14:489-505, 2014

28. Taylor RS, Van Buyten JP, Buchser E: Spinal cord stimulation for chronic back and leg pain and failed back surgery syndrome: A systematic review and analysis of prognostic factors. Spine (Phila Pa 1976) 30:152-160, 2005 
29. Turner J, Hollingworth W, Comstock B, Deyo R: Spinal cord stimulation for failed back surgery syndrome: Outcomes in a workers' compensation setting. Pain 148:14-25, 2010

30. Van Buyten JP: Neurostimulation for chronic neuropathic back pain in failed back surgery syndrome. J Pain Symptom Manage 31:25-29, 2006

31. Vonhogen LH, Vancamp T, Vanneste S, Pollet W, Dirksen R, Bakker P, Mestrom I, van de Looij T, Plazier M, de Ridder D: Percutaneously implanted plates in failed back surgery syndrome (FBSS). Neuromodulation 14:319-324, 2011 